\title{
Pelvic Inflammatory Disease: An Evidence-Based Guideline
}

\author{
Irami Araújo-Neto루, Tarciso Bruno Montenegro Sampaio², Amália Cinthia Meneses do Rêgo ${ }^{3}$ and Irami Araújo- \\ Filho $^{4 *}$
}

${ }^{1}$ Undergraduate Student of the Medicine Course, Potiguar University (UnP), Brazil

${ }^{2}$ Department of Biology, Potiguar University (UnP), Brazil

${ }^{3}$ Post Graduation Program in Biotechnology, Potiguar University (UnP), Brazil

${ }^{4}$ Full Professor of the Post Graduate Program in Biotechnology at Potiguar University, Brazil

Received: : March 12, 2018; Published: 盋 March 22, 2018

*Corresponding author: Irami Araújo Filho, Department of Surgery, Federal University of Rio Grande do Norte. Full Professor, Department of Surgery, Potiguar University. PhD in Health Science, Natal - RN, 59020-650, Brazil, Tel: 5584 988760206; Email: irami.filho@uol.com.br

\begin{abstract}
A group of medical students and researchers from the Northeastern Region of Brazil produced this guideline, in order to provide doctors or health teams who work in the so-called Brazilian Primary Care Programs as well as those professionals working in the most remote interiors of the country. Guideline-based on current scientific evidence and adapted to the Brazilian reality, to help in the treatment and recovery of the health of women affected by the pelvic inflammatory disease, especially those young, adolescent patients with no access to information.
\end{abstract}

Keywords: Pelvic inflammatory diseases; Pelvic diseases; Guideline; Sexually transmitted disease; Bacterial infections; Therapies

\section{Introduction}

Pelvic inflammatory disease (PID) refers to the acute infection of the upper genital tract structures in women, involving any of the following structures of the uterus, ovaries and fallopian tubes, or in an association. This pathology is often accompanied by involvement of pelvic organs neighbors [1-3]. By definition, the DIP is a community-acquired infection initiated by a sexually transmitted agent, distinguishing the pelvic infections caused by medical procedures, pregnancy, and another abdominal processes primary [4].

\section{Methods}

We reviewed the literature in PUBMED, SCOPUS, WEB OF SCIENCE and EMBASE, using the descriptors below keywords, selecting the main scientific evidence chosen by peers and oriented by professionals in the field, involving randomized clinical studies, systematic reviews, meta-analyses and the main guidelines found in literature over the last five years, for the construction of the present revision, which became the guideline based on scientific evidence and adapted the working conditions in the health of the professionals of the Northeast of Brazil.

\section{Results and Discussion}

Most cases of PID are mild-to-moderate uncomplicated forms that can be treated as outpatients. Apart from Chlamydia trachomatis and Neisseria gonorrhoeae, other pathogens such as bacterial vaginosis and Mycoplasma genitalium associated bacteria are playing a significant role in PID and thus must be reckoned with. Moreover, gonococci have increasingly become resistant to the majority of antibiotics [5]. This has led to a universal recommendation to treat $\mathrm{N}$. gonorrhoeae infections with ceftriaxone. The pelvic inflammatory disease is caused by the spread of organisms from the vagina and cervix to the upper genital tract. The patient may have a life-threatening illness or may remain asymptomatic $[6,7]$. The disease may be caused by sexually transmitted no sexually or organisms. Polymicrobial infections are the rule. Therapy requires antibiotic agents with broad coverage of 
aerobic and anaerobic organisms, including Chlamydia trachomatis and Neisseria gonorrhoeae [8].

\section{Pathogenesis}

The vaginal flora of women includes a variety of potentially pathogenic bacteria. Among these are the species of Staphylococcus, Streptococcus, Enterobacteriaceae (Klebsiella spp, Escherichia coli, Proteus spp.), and a variety of anaerobic. In comparison with the Lactobacillus acidophilus producers of hydrogen peroxide, these organisms are present in low numbers and present fluctuation due to several factors, including hormonal changes, contraceptive method, activity and others yet unknown $[2-3,9]$. The complete change of the vaginal ecosystem can occur when anaerobic bacteria take predominance over the strains of Lactobacilli. This condition is known as bacterial vaginosis, which is not associated with an inflammatory response.

The intermediate situation in which Lactobacilli and anaerobic hydrogen peroxide producers coexist in approximately equal numbers is called a transitional flora [10]. The endocervical channel functions as a protective barrier of the upper genital tract, normally sterile, the bodies of the vaginal ecosystem occur dynamically [11]. An imbalance of this barrier provides access to vaginal bacteria to the upper genital organs through canaliculus channels, infecting the endometrium, endosalpinges, ovarian cortex, peritoneum and pelvic your underlying stroma. This is the clinical entity called pelvic inflammatory disease (PID) [12].

DIP patients may present with clinical disease at any point along the genital tract (endometrium with normal tubes, ovaries, and peritoneum) [1,2]. More than $75 \%$ of cases occur within seven days of menstruation, the time during which the quality of the cervical mucus promotes vaginal organisms rise [13]. Epidemiological data suggest that all sexually transmitted and cause are histological and structural changes in the endocervical barrier. The most common microorganisms in this process are the pathogenic Neisseria gonorrhoeae and Chlamydia trachomatis $[1,14]$.

\section{Risk factors}

Women without active sex life have no risk of acquiring PID and the longtime monogamous women rarely develop. On the other hand, women with multiple partners are at higher risk. The condition, age, and race DIP are other important risk factors. The frequency is also affected by contraceptive method used [15]. The evaluation of the patient for risk factors in contracting sexually transmitted diseases and those that increase the likelihood of a DIP is very important $[1-4,16]$.

Multiple partners: PID risk has increased 3.4 times by four or more sex partners during the last six months, and 3.2 times a sexual relationship with a single partner six or more times a week [1].

The situation of the partner: Abouthalf of men with gonococcal or chlamydial urethritis is asymptomatic. Have a symptom (dysuria, urethral discharge) in the male partner increases the risk of the woman PID [2].

Age: The PID occurs in higher frequency between 15 and 25 years of age; the incidence in women over 35 years of age is just one-seventh of that in younger women [17].

PID preview: PID prior to subsequent episodes raised the risk by a factor of 2.3 in a report, and approximately one in every four women will suffer recurrence with PID.

Barrier Contraception: Barrier contraception protects against PID. Condoms are the most effective, avoiding $50 \%$ of gonococcal and chlamydial infections endocervical specimens the addition of 9 -nonoxynol gives protection over $25 \%[2,3]$.

Oral contraceptives: Oral contraceptives (OC) have a complex interaction with DIP. Several studies have shown that using CO almost doubles the prevalence of chlamydia and gonococcal infection of the cervix. However, using CO has traditionally been associated with a $50 \%$ reduction in the risk of PID [18].

Intrauterine device (IUD) and tubal sterilization: Modern intrauterine devices are associated with reduced risk of PID. The risk of PID is essentially limited to the first three weeks after the insertion of the IUD and is unusual. No evidence suggests that IUDs should be removed from women diagnosed with acute PID $[4,5,19]$. Tubal ligation can protect the distal fallopian tubes of involvement, but the clinical syndrome of PID is otherwise affected [6]. Other the PID occurs more commonly among African Americans. It may be more common among women who use vaginal shower [2-5].

\section{Clinical manifestations}

The clinical diagnosis of PID is imprecise. Abdominal pain in lower belly is the cardinal symptom in women with PID, although the character of the pain can be very subtle. The recent emergence of worsening pain during intercourse or with sudden movement can be the only symptom of the PID; the beginning of pain during or shortly after the period is particularly suggestive. The abdominal pain is usually bilateral and rarely lasting more than two weeks [20-22]. Abnormal uterine bleeding occurs in one-third or more of patients with PID. The presence of a new, urethritis vaginal discharge, fever and chills may result but are not sensitive or specific for the diagnosis. The presence of PID is less likely if there are symptoms related to the intestines or urinary tract of predominant form $[4,5]$. Although it is rare to have PID during pregnancy, the infection can occur in the first 12 weeks of pregnancy before the advent of the mucous plug and deciduous seal outside the uterus protect bacteria ascending pregnant [23].

On physical examination, only $50 \%$ of patients with PID have a fever. The abdominal examination reveals increased soreness of diffuse mode higher in lower quadrants, which may or may not be symmetrical [24]. The pelvic exam, the finding of a purulent endocervical discharge and/or sudden movement of cervical and 
annex with increased sensitivity painful two-hand examination is strongly suggestive of PID. Rectovaginal examination combined should show the uterus and attachments as the focus of pain. Other diagnoses should be considered if the uterine and adnexal pain are not prominent [25].

Peri-hepatitis: Peri-hepatitis (Fitz-Hugh-Curtis Syndrome) was initially associated with salpingitis gonococcal urethritis in 1920 and later the Chlamydia trachomatis ${ }^{12}$. It is characterized by infection of the liver capsule and peritoneal surface of the upper right quadrant before, with the involvement of minimal hepatic stroma. It manifests as a fibrinous exudate and purulent in the acute phase, affecting most prominently the previous faces of the liver (not hepatic parenchyma) [2-6,26]. The symptoms are sudden onset of intense pain in the right upper quadrant of the abdomen with a pleuritic component, sometimes referred to in the right shoulder. The severity of pain that can mask the diagnosis of PID and concerns about acute cholecystitis. Transaminases are usually normal or only slightly elevated [27].

\section{Differential diagnosis}

Laparoscopy: Laparoscopy has substantial value in the confirmation of the diagnosis of PID but is not sensitive enough to be considered a gold standard of diagnosis. We recommend laparoscopy in the following clinical pictures [2-5,28]:

i. The symptomatic patient with high suspicion of a diagnosed o segment competitor (usually appendicitis, etc.);

ii. Woman with the acute disease, which did not respond to outpatient treatment to PID;

iii. Any patient is the clearly improving after approximately $72 \mathrm{~h}$ of hospital treatment to PID.

iv. Is required to obtain consent for performing laparoscopy in patients with an indication for the procedure. Endometrial biopsy is not recommended in these cases unless the procedure is part of a Protocol.

\section{Diagnostic criteria}

The index of suspicion for the clinical diagnosis of PID needs to be raised, especially in teenage women, even as it denies the achievement of sexual activity. The approach advocated for the diagnosis of PID is multifaceted [1].

A minimum set of clinical criteria has been recommended by the Centers for Disease Control and Prevention (CDC) for the empirical treatment of PID, including pain on palpation cervical or uterine or sensitivity of attachments in the presence of abdominal or pelvic pain, during the examination physical. The following additional criteria can also be used to confirm the clinical diagnosis of PID [1-4]:

a. $\quad$ Oral temperature $>101^{\circ} \mathrm{F}\left(38.3^{\circ} \mathrm{C}>\right)$;

b. Mucopurulent cervical discharge or abnormal vaginal; c. The presence of abundant numbers of white blood cells (leukocytes) on saline microscopy of vaginal secretions;

d. Elevated erythrocyte sedimentation rate;

e. High Proteína C-reactive;

ii. Patients with pelvic pain or any of the following clinical findings are currently considered "confirmed" cases:

iii. Acute or chronic acute salpingitis or endometritis in histological evaluation of biopsy;

iv. Demonstrates integration N.gonorrhoeae or C. trachomatis in the genital tract;

v. Diagnostic laparoscopy or laparotomy for PID;

vi. Isolation of pathogenic bacteria from a bacterial specimen from the upper genital tract;

vii. Pelvic inflammatory purulent fluid without another source;

viii. The Centers for disease control and prevention (CDC) has issued guidelines for the "definitive" diagnosis of symptomatic patients PID. In the presence of one or more of the following three criteria are needed for the diagnosis:

ix. Histological evidence of endometritis in a biopsy;

$\mathrm{x}$. A $\mathrm{t}$ is image technique, revealing thick liquid/uterine tubes with or without free pelvic fluid or tubo-ovarian complex compromised;

xi. Laparoscopic abnormalities consistent with PID (erythema, edema, tubal adhesions; purulent exudate or purulent fluid in Douglas bag Fund; fimbrias freak);

\section{Staying}

a) Stage 1 - Endometritis and acute salpingitis without peritonitis;

b) Stage 2 - Salpingitis with peritonitis;

c) Stage 3 - Acute salpingitis with tubal occlusion or impairment

d) tube-ovarian. Complete abscess;

e) Stage 4 - Broken tube-ovarian abscess. Purulent secretion in the cavity.

Acute PID is difficult to diagnose due to the diversity of signals and symptoms. Delay in diagnosis and treatment probably contributes to the sequelae of the upper reproductive tract. The exuberance of symptoms in PID is not always observed. In many cases, the symptomatology is poor, with only mild bleeding occurring translates to initial endometritis, eventually associated with symptoms of cervicitis 
or urethritis. In such cases, screening for chlamydia is critical to diagnosis. Data on epidemiology are very important and should be observed during anamnesis and physical examination [1,29].

\section{Differential diagnosis}

In addition to PID, the differential diagnosis of lower abdominal pain in a young woman includes the following conditions $[3-4,30]$ :

a. Gastrointestinal: appendicitis, cholecystitis, constipation, gastroenteritis, inflammatory bowel disease, perforated peptic ulcer, acute pancreatitis, Meckel diverticulitis, appendix tumor; intestinal obstruction, flanges;

b. Urologic: cystitis, pyelonephritis, renal lithiasis, distal ureteral lithiasis, urethritis;

c. Obstetric/Gynecological: dysmenorrhea, ectopic pregnancy, intrauterine device infection or rejection, pregnancy complications, ovarian cyst, ovary torsion, ovarian tumor, endometriosis.

\section{Diagnostic tests}

The non-invasive diagnostic tests to PID include general laboratory studies looking for signs of inflammation, and culture tests cervical or vaginal secretions microscopy and imaging tests [25-28].

We recommend the following laboratory tests:

a. Pregnancy test;

b. Microscopic examination of vaginal discharge in saline solution;

c. Complete blood count;

d. Nucleic acid amplification tests for C. trachomatis and N. gonorrhoeae;

e. The urine test;

f. C-reactive protein (optional);

g. HIV tests;

h. Surface antigen and Hepatitis B surface antibodies;

i. $\quad$ Test for syphilis.

\section{Radiology}

Ultrasound findings inconclusive studies observed in patients with PID, so, we recommend a transvaginal approach instead of transabdominal [15-18]. Uterine tubes filled with liquid observed by ultrasound are useful to support the clinical and radiological diagnosis of PID. However, the absence of amendments does not diminish the likelihood of PID and should not be used as a reason for delaying treatment. Reserve money for the patients with acute disease with DIP in a pelvic abscess is one of the hypotheses to be confirmed [4-8,31].
The most specific diagnostic tools include endometrial biopsy with histopathological evidence of endometritis, transvaginal USG or MRI magnetic resonance imaging showing tube-ovarian or doppler complex suggestive of infection pelvic abnormalities and consistent with PID. Laparoscopy, despite being considered the gold standard for the diagnosis of PID, has not been widely used, due to the high cost and morbidity associated. Another fact to be considered is that, in the initial stages, when the inflammatory process is restricted to tubal lumen, laparoscopic vision may result in a false-negative diagnosis [20-23].

\section{Treatment}

Physicians should maintain a low threshold of suspicion for the diagnosis of PID. Acute DIP should be considered in sexually active young women complaining of pain in lower abdomen, pelvic tenderness (pain cervical, uterine sensitivity to touch or pain in attachments) [8-10,17]. Response to treatment is based on both short-term and long-term results. The clinical cure is defined as "important" or "improves" the signs and/or symptoms of DIP. Microbiological cure is defined as eradication of $N$. gonorrhoeae or C. trachomatis, if present at baseline $[25,26]$.

\section{Initial evaluation}

Outpatient treatment applies to women clinical examination, abdominal and gynecological examination without signs of pelviperitonitis, and are not included in the criteria for hospital treatment $[2,3,32]$.

Criteria for hospitalization and antibiotic intravenous therapy:

i. If surgical emergencies cannot be ruled out;

ii. Severe pain, nausea, and vomiting or high fever;

iii. Tube-ovarian abscess;

iv. Inadequate response to outpatient treatment;

v. Inability to follow outpatient treatment;

vi. Pregnancy.

\section{General measures}

i. Rest and sexual abstinence during treatment;

ii. Convene partners for clinical evaluation (advise that men with

iii. gonococcal infection or chlamydia are usually symptomatic);

iv. Guidance on the risks of new episodes of PID and other STDs;

v. Avoid unprotected sex;

vi. Offer and collect, after counseling, serology for syllables,

vii. Hepatitis B and HIV. 
viii. Rest, hydration and symptomatic treatment (analgesics, antipyretics and non-hormonal anti-inflammatory drugs).

\section{Hospital treatment versus outpatient treatment}

There is a persistent tendency to outpatient treatment of PID in 50-75\% of women. Clinical trials data support such an approach in patients with mild or moderate PID. In the $25-30 \%$, where the presence of severe PID, and when associated with other complicating factors, there is indication for hospitalization [1-5, 3335].

a. Inpatient therapy with cefoxitin intravenously (2 g-6/6:00) plus doxycycline (100mg-2 x/day-14 days);

b. Outpatient therapy with a single intramuscular dose of cefoxitin (2g), plus a single oral dose of Probenecid (1g) plus oral doxycycline (100 mg-2 x/day-14 days).

\section{Indications for hospital treatment/Hospitalization}

Indications for hospitalization and use of parenteral antibiotics include [12-14]:

a. Pregnancy;

b. Lack of response or intolerance to oral medications;

c. Non-adherence to outpatient treatment;

d. Inability to take oral medications due to nausea and vomiting;

e. Severe clinical disease (high fever, nausea, vomiting, severe abdominal pain);

f. PID complicated with pelvic abscess (including ovarian tube abscess);

g. Possible need for surgical intervention or diagnostic exploration to rule out or confirm other etiologies (e.g., appendicitis).

There is no scientific evidence to suggest that the elderly or patients with immunodeficiency virus should be considered criteria for hospitalization.

\section{Antibiotic therapy}

General approach-the antibiotics selected should have activity against Neisseria gonorrhoeae and Chlamydia trachomatis, is the most prevalent in the PID. Gonococcal resistance to medicinesfrom 2007, CDC guidelines state that fluoroquinolones no longer recommended as therapy for PID if $N$. gonorrhoeae is the pathogen proved or suspect [4-8]. Inpatient Therapy - Patients with severe PID should be hospitalized and treated with parenteral antibiotics. The indications for hospitalization are discussed above.

First-line Therapies: For patients with severe PID, the CDC recommends one of the following parenteral regimens that result in clinical cure of acute disease in more than $90 \%$ of cases ${ }^{10}$ : a. Cefoxitin (2g-EV-6/6h) or Cefotetan (2g-EV-12/12h) associated with Doxycycline (100mg - orally-12/12h).

b. Clindamycin (900mg-EV-8/8h) associated with Gentamicin $(2 \mathrm{mg} / \mathrm{kg})$ followed by a maintenance dose $(1,5 \mathrm{mg} /$ $\mathrm{kg}$ ) every $8 \mathrm{~h}$. Unique daily intravenous gentamicin can be replaced by three times daily dosing.

b) Antiemetic drugs and antipyretics should be offered to those patients who are symptomatic.

c) The patients with pelvic abscess should also receive oral Clindamycin 450mg every $6 \mathrm{~h}$ or Metronidazole 500mg every $8 \mathrm{~h}$ for a total of 14 days plus Doxycycline. The management of the patient with complicated PID with ovarian tube abscess is discussed separately.

d) Alternative schemes-limited data are available on the following schemes, which are considered "alternatives" by the CDC:

a. Ampicillin-sulbactam (3g-EV-6/6h), Doxycycline (100mg twice a day) led to a similar rate of clinical cure compared to Cefoxitin (2g-6/6h) plus Doxycycline (100mg-2x/ day) among women hospitalized with PID (86 x 89\%).

e) Outpatient Therapy - Patients with mild or moderate PID are appropriate candidates for oral therapy once the clinical results are equivalent with oral or parenteral therapy.

f) First line schemes -The CDC recommends any of the following outpatient regimens, with or without metronidazole (500mg- 2x/day -14 days):

a. Ceftriaxone (250 mg-IM - single dose) plus Doxycycline (100mg-oral-2 x/day-14 days);

b. Cefoxitin (2g-IM - single dose) associated with Probenecid (1g-orally at a single dose) plus Doxycycline (100mg orally-2x/ day-14 days);

c. Other parenteral third-generation cephalosporins, such as Cefotaxime (1g-IM-single dose) or Cefoxitin (1g-IM-single dose) plus Doxycycline (100mg orally twice a day-14 days);

g) The cephalosporin, ceftriaxone has better overall activity against gonococcal infection. It is preferred if ceftriaxone plus doxycycline in patients with mild to moderate renal insufficiency. Metronidazole should be added for patients with Trichomonas vaginalis or those women with recent history of invasive uterine manipulation [30-33].

Duration of treatment: Great duration of treatment is unknown. Most studies have used 14 days of therapy and your duration was kept in the STD 2010 CDC recommendations [1-6].

Patients with a diagnosis of acute PID should undergo counseling of safe sexual practices. 


\section{Surgery}

i. Clinical treatment failure;

ii. Presence of pelvic mass that persists or increases, despite the clinical treatment;

iii. Suspected rupture of tube-ovarian abscess;

iv. Hemoperitoneum;

v. Douglas sack bottom abscess;

vi. Conservative surgeries are preferable and allow 10 to $15 \%$ rate postoperative fertility;

vii. Hysterectomy and annexectomy for patients with complete offspring;

viii. Bankruptcy of drug therapy is generally associated with abscesso which can be conducted with transabdominal drainage or transvaginal guided by CT or laparoscopy.

\section{Sexual partners}

Male partners of women with PID should be examined and treated if they had sexual contact with the patient during the last 60 days before onset of symptoms, regardless of the results of the diagnostic tests. Assessment and treatment of sexual partner is essential to reduce the risk of reinfection. Therapeutic schemes should include antibiotics with activity against Neisseria gonorrhoeae and Chlamydia trachomatis, such as Ceftriaxone (250mg) subcutaneously plus Azithromycin $1 \mathrm{~g}$ orally as a single dose or Doxycycline (100mg) orally twice a day for seven days [2833].

\section{Follow-Up}

a) Review with 72 hours to evaluate clinical improvement. Admit to hospital if there is no substantial improvement.

b) Consider review after 4 weeks after therapy to evaluate adequate adherence and response to treatment;

c) Conclude screening and treatment of partners;

d) Discuss potential sequelae of PID.

e) Cure tests are required if symptoms persist after treatment;

f) Antibiotic resistance is considered (particularly in the case of gonococcus);

g) Poor adherence to treatment is suspected or therapy is not tolerated;

h) The possibility of reinfection $[1,2]$.

i) Complications

j) PID has high morbidity;

k) Tubal infertility in $15 \%, 35 \%$ and $55 \%$ of women after 1
2 and 3 episodes of PID respectively;

l) Tube-ovarian abscess develops in approximately 7-16\%;

m) Recurrent infection occurs in 20-25\%;

n) Repeated episodes of PID are associated with an increase of 4 to times of permanent tubal damage;

o) About $10 \%$ of those who conceive have ectopic pregnancy;

p) Chronic pelvic pain in $20 \%$ related to the formation of adhesions, chronic salpingitis or recurrent infections;

q) Patient Monitoring;

r) Careful observation of clinical status, particularly for fever, peritonitis and leukocytosis;

s) Accompany size and position of adnexal abscess with USG $[2-6,35]$.

\section{Hydro salpingitis}

After treated the DIP, the damaged fallopian tubes can become blocked, is full of sterile liquid and become bigger. Damage to the fallopian tube or adhesion surgery earlier can also result in hydrosalpinges. The hydrosalpinges may be associated with pain or may be asymptomatic except for tubal infertility factor $[3,4]$.

\section{Chronic pelvic pain}

Chronic pelvic pain is defined as pain or menstrual menstrual with a minimum duration of six months, that occurs below the navel and is severe enough to cause functional disability. Although the precise etiology is unknown, pain can result from scarring and adhesions that develop from the inflammation related to the infectious process [2].

\section{Infertility}

DIP both symptomatic as asymptomatic can cause permanent damage to the fallopian tubes, especially in endosalpingitis. Changes in the fallopian tube, including the loss of the ciliary action, fibrosis and occlusion, tubal infertility promote [1-3,35].

\section{Conclusion}

PID is a condition of high prevalence and associated with too significant morbimortality. It's about the significant cause of infertility, ectopic pregnancy, and chronic pelvic pain. The most common etiologic agent is Chlamydia trachomatis and about $70 \%$ of the women infected by this agent are asymptomatic. The diagnosis is clinical, presenting predictive value between 65 and 90\%. Your Suspicion Followed for rapid diagnosis and early establishment treatment is the best way to preserve the reproductive future of women.

\section{References}

1. Brunham RC, Gottlieb SL, Paavonen J (2015) Pelvic inflammatory disease. N Engl J Med 372(21): 2039-2048. 
2. Ross J D (2013) Pelvic inflammatory disease. BMJ Clin Evid 11: 1606.

3. Ford GW, Decker CF (2016) Pelvic inflammatory disease. Dis 62(8): 301305.

4. Brun J L, Graesslin O, Fauconnier A, Verdon R, Agostini A, et al. (2016) Updated French guidelines for diagnosis and management of pelvic inflammatory disease. Int J Gynaecol Obstet 134(2): 121-125.

5. Bugg CW, Taira T (2016) Pelvic Inflammatory Disease: Diagnosis And Treatment In The Emergency Department. Emerg Med Pract 18(12): $1-24$.

6. Jensen JS, Cusini M, Gomberg M, Moi H (2016) European guideline on Mycoplasma genitalium infections. J Eur Acad Dermatol Venereol 30(10): 1650-1656.

7. Ross J, Judlin P, Jensen J, International Union against sexually transmitted infections (2012) European guideline for the management of pelvic inflammatory disease. Int J STD AIDS 25(1): 1-7.

8. Haggerty CL, Totten PA, Tang G, Astete SG, Ferris MJ, et al. (2016) Identification of novel microbes associated with pelvic inflammatory disease and infertility. Sex Transm Infect 92(6): 441-446.

9. Alcaraz I, Dupin N, Janier M, Derancourt C, Milpied B, Bertolotti (2016) [Mycoplasma genitalium]. Ann Dermatol Venereol 143(11): 718-719.

10. Pérez-López FR, Ornat L, Ceausu I, Depypere H, Erel CT, et al. (2014) EMAS position statement: management of uterine fibroids. Maturitas 79(1): 106-116.

11. Caddy S, Yudin MH, Hakim J, Money DM (2014) Best practices to minimize risk of infection with intrauterine device insertion. J Obstet Gynaecol Can 36(3): 266-274.

12. Savaris RF, Fuhrich DG, Duarte RV, Franik S, Ross J (2017) Antibiotic therapy for pelvic inflammatory disease. Cochrane Database Syst Rev 4: CD010285.

13. Han C, Wu W, Fan A, Wang Y, Zhang H, et al. (2015) Diagnostic and therapeutic advancements for aerobic vaginitis. Arch Gynecol Obstet 291(2): 251-257.

14. Wiesenfeld HC (2017) Screening for Chlamydia trachomatis Infections in Women. N Engl J Med 376(8): 765-773.

15. Zakher B, Cantor AG, Pappas M, Daeges M, Nelson HD (2014) Screening for gonorrhea and Chlamydia: a systematic review for the US Preventive Services Task Force. Ann Intern Med 161(12): 884-893.

16. Price MJ, Ades AE, Welton NJ, Simms I, Macleod J, et al. (2016) Proportion of Pelvic Inflammatory Disease Cases Caused by Chlamydia trachomatis: Consistent Picture From Different Methods. J Infect Dis 214(4): 617-624.

17. Tsevat DG, Wiesenfeld HC, Parks C, Peipert J F (2017) Sexually transmitted diseases and infertility. Am J Obstet Gynecol 216(1): 1-9.

18. Peng T, Dong L, Zhu Z, Cui J, Li Q, et al. (2016) CT-guided Drainage of Deep Pelvic Abscesses via a Percutaneous Presacral Space Approach: A Clinical Report and Review of the Literature. Acad Radiol (12): 15531558.

19. Spain J, Rheinboldt M (2017) MDCT of pelvic inflammatory disease: a review of the pathophysiology, gamut of imaging findings, and treatment. Emerg Radiol 24(1): 87-93.
20. Revzin MV, Mathur M, Dave HB, Macer ML, Spektor M (2016) Pelvic Inflammatory Disease: Multimodality Imaging Approach with ClinicalPathologic Correlation. Radiographics 36(5): 1579-1596.

21. Price MJ, Ades AE, Soldan K, Welton NJ, Macleod J, et al. (2016) The natural history of Chlamydia trachomatis infection in women: a multiparameter evidence synthesis. Health Technol Assess 20(22): 1-250.

22. Zhu H, Shen Z, Luo H, Zhang W, Zhu X (2016) Chlamydia Trachomatis Infection-Associated Risk of Cervical Cancer: A Meta-Analysis. Medicine 95(13): 3077.

23. Speer LM, Mushkbar S, Erbele T (2016) Chronic Pelvic Pain in Women. Am Fam Physician 93(5): 380-387.

24. Lancaster JW, Mahoney MV, Mandal S, Lawrence KR (2015) Update on Treatment Options for Gonococcal Infections. Pharmacotherapy 35(9): 856-868.

25. Cho HW, Koo YJ, Min KJ, Hong JH, Lee JK (2017) Pelvic Inflammatory Disease in Virgin Women With Tubo-ovarian Abscess: A Single-Center Experience and Literature Review. J Pediatr Adolesc Gynecol 30(2): 203208.

26. Duarte R, Fuhrich D, Ross JD (2015) A review of antibiotic therapy for pelvic inflammatory disease. Int J Antimicrob Agents 46(3): 272-277.

27. Polena V, Huchon C, Varas Ramos C, Rouzier R, Dumont A, et al. (2015) Non-invasive tools for the diagnosis of potentially life-threatening gynaecological emergencies: a systematic review. PLoS One 10(2): e0114189.

28. Hafner LM (2015) Pathogenesis of fallopian tube damage caused by Chlamydia trachomatis infections. Contraception 92(2): 108-115.

29. Greydanus DE, Dodich C (2015) Pelvic inflammatory disease in the adolescent: a poignant, perplexing, potentially preventable problem for patients and physicians. Curr Opin Pediatr 27(1): 92-99.

30. Cheong YC, Smotra G, Williams AC (2014) Non-surgical interventions for the management of chronic pelvic pain. Cochrane Database Syst Rev (3): CD008797.

31. Lis R, Rowhani Rahbar A, Manhart LE (2015) Mycoplasma genitalium infection and female reproductive tract disease: a meta-analysis. Clin Infect Dis 61(3): 418-426.

32. Yang SF, Wu TF, Tsai HT, Lin LY, Wang PH (2014) New markers in pelvic inflammatory disease. Clin Chim Acta 431: 118-124.

33. Sharma H, Tal R, Clark N A, Segars J H (2014) Microbiota and pelvic inflammatory disease. Semin Reprod Med 32(1): 43-49.

34. Mitchell C, Prabhu M (2013) Pelvic inflammatory disease: current concepts in pathogenesis, diagnosis and treatment. Infect Dis Clin North Am 27(4): 793-809.

35. Trent M (2013) Status of adolescent pelvic inflammatory disease management in the United States. Curr Opin Obstet Gynecol 25(5): 350356. 
(C) (P) This work is licensed under Creative To Submit Your Article Click Here: Submit Article

DOI: 10.32474/IGWHC.2018.01.000120

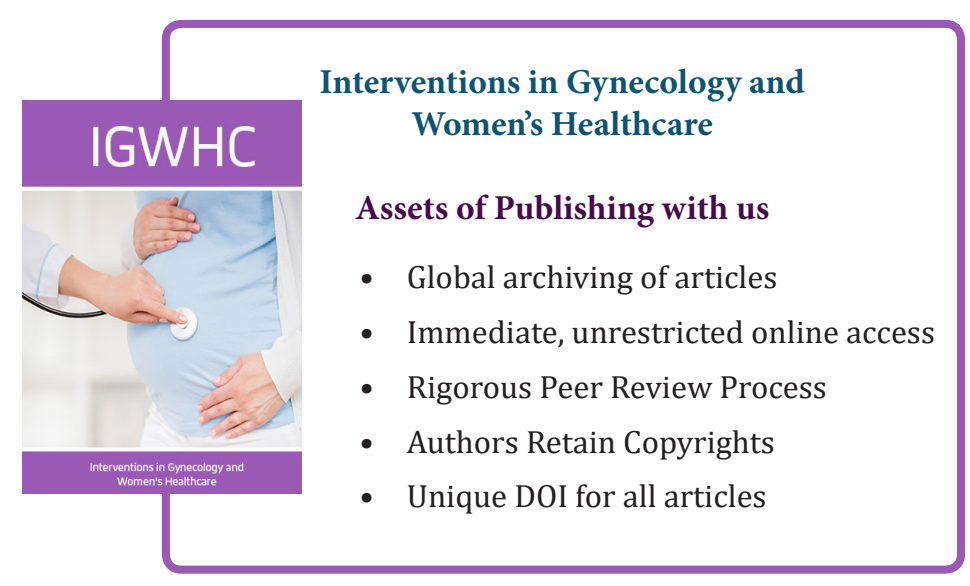

